\title{
Polynomial Reproduction of Vector Subdivision Schemes
}

\author{
Y. F. Shen, ${ }^{1,2}$ D. H. Yuan, ${ }^{3}$ and S. Z. Yang ${ }^{1}$ \\ ${ }^{1}$ Department of Mathematics, Shantou University, Shantou, Guangdong 515063, China \\ ${ }^{2}$ Department of Mathematics, Dezhou University, Dezhou 253023, China \\ ${ }^{3}$ Department of Mathematics, Hanshan Normal University, Chaozhou, Guangdong 521041, China
}

Correspondence should be addressed to S. Z. Yang; szyang@stu.edu.cn

Received 1 April 2014; Accepted 25 May 2014; Published 7 July 2014

Academic Editor: Wenchang Sun

Copyright (C) 2014 Y. F. Shen et al. This is an open access article distributed under the Creative Commons Attribution License, which permits unrestricted use, distribution, and reproduction in any medium, provided the original work is properly cited.

\begin{abstract}
We discuss the polynomial reproduction of vector subdivision schemes with general integer dilation $m \geq 2$. We first present a simple algebraic condition for polynomial reproduction of such schemes with standard subdivision symbol. We then extend it to general subdivision symbol satisfying certain order of sum rules. We also illustrate our results with several examples. Our results show that such kind of scheme can produce exactly the same scalar polynomial from which the data is sampled by convolving with a finite nonzero sequence of vectors.
\end{abstract}

\section{Introduction}

Subdivision schemes are efficient algorithmic methods in approximation theory, computer aid geometric design, and wavelet construction; see $[1,2]$ and references therein. The subdivision scheme is called vector subdivision scheme if the corresponding subdivision symbol is a matrix of trigonometric polynomial. Note that the vector subdivision scheme plays an important role in multiwavelet and multichannel wavelets analysis; see [3-5] and references therein. In this paper we would like to derive simple algebraic conditions on the subdivision symbol that allow us to determine the degree of its polynomial reproduction, which is different from polynomial generalization.

The polynomial generalization is the capability of vector subdivision scheme to generate the full space of scalar polynomials with desired degree. This property is equivalent to approximation order (defined in $L^{2}$-norm), accuracy, and sum rulers; see $[6,7]$. The so-called polynomial reproduction is the capability of vector subdivision scheme to produce exactly the same scalar polynomial $p(x)$ from which the data is sampled by convolving with a special sequence of vectors $\mathbf{h}_{0}=\left\{\mathbf{h}_{0, j} \in \mathbb{R}^{s}, j \in \mathbb{S}\right\}$,

$$
\mathbf{d}_{0, k}^{T}:=\left(p * \mathbf{h}_{0}\right)(k)=\sum_{j \in \mathbb{Z}} p(k-j) \mathbf{h}_{0, j}, \quad k \in \mathbb{Z}
$$

where $\mathbb{S}$ is some finite index set containing $0, *$ denotes convolution operator, and $T$ denotes transpose operator.

There are many valuable works that were done when scalar subdivision scheme was considered [8-11]. Hormann and Sabin [8] presented some algebraic conditions which can be used to determine the degree of polynomial reproduction for a family of schemes. Polynomial reproduction was studied by Dyn et al. [9] for arbitrary prime and dual binary schemes and extended to univariate subdivision scheme of any arity by Conti and Hormann [10]. Further, Charina and Conti [11] yielded simple algebraic conditions on the subdivision symbol for the multivariate setting of scalar subdivision with dilation matrix $m I,|m|>1$; Charina and Romani [12] extended to the general expanding dilation matrix $M \in \mathbb{Z}^{\text {s×s }}$ case. Motivated by all these works, we discuss the property of polynomial reproduction for vector subdivision schemes.

The remainder of this paper is organized as follows. Section 2 sets the notations concerning vector subdivision scheme with general integer dilation $m \geq 2$ and stresses the connection of convergence between the vector subdivision scheme and traditional cascade algorithm. In Section 3, we provide algebraic tools for determining the degree of polynomial reproduction of the vector subdivision scheme with standard subdivision symbol; basing on two scale similarity transform (TST), we also establish the relationship of vector subdivision schemes between general subdivision symbol 
and the standard subdivision symbol. In Section 4, several examples are provided to illustrate our results.

\section{Background and Notation}

A vector subdivision scheme $\mathbf{S}_{P, \mathbf{h}_{0}, \Phi_{0}}$ with integer dilation $m \geq 2$ is given by a finitely supported matrix sequence $P=\left\{P_{k} \in \mathbb{R}^{s \times s}, k \in \mathbb{Z}\right\}$ (so-called subdivision mask), a constant column vector $\mathbf{h}_{0}=\left\{\mathbf{h}_{0, j} \in \mathbb{R}^{s}, j \in \mathbb{S}\right\}$, and an initial function $\Phi_{0}(x)$. The constant vector $\mathbf{h}_{0}$ and the initial function $\Phi_{0}$ are determined by $P$ and will be given by (39) or (55) in the next section. The symbols of $\mathbf{h}_{0}$ and $P$ (denoted by $\mathbf{h}_{0}(z)$ and $P(z)$, resp.) are given by the Laurent polynomials

$$
\mathbf{h}_{0}(z)=\sum_{j \in \mathbb{S}} \mathbf{h}_{0, j} z^{j}, \quad P(z)=\sum_{k \in \mathbb{Z}} P_{k} z^{k}
$$

respectively.

In this paper, we use two vector spaces of Laurent polynomial, $\mathfrak{Q}\left(\mathbb{R}^{s}\right)$ and $\mathbf{\Omega}^{\prime}\left(\mathbb{R}^{s}\right)$. We say $\mathbf{h}_{0}(z) \in \mathfrak{Q}\left(\mathbb{R}^{s}\right)$, if

$$
\mathbf{h}_{0}^{T}(z)=\mathbf{h}^{T} A(z)=\mathbf{h}^{T}\left[\begin{array}{cc}
1 & \sum_{j \in \mathbb{S}} \mathbf{r}_{j} z^{j} \\
\mathbf{0} & I_{s-1}
\end{array}\right],
$$

with some $\mathbf{h} \in \mathbb{R}^{s}$ and $\mathbf{h} \neq \mathbf{0}$, where $\left\{\mathbf{r}_{j}, j \in \mathbb{S}\right\}$ is some finite $1 \times(s-1)$-vector sequence and $I_{s-1}$ is the $(s-1) \times(s-1)$ identity matrix. Similarly, we say $\mathbf{h}_{0}(z)$ belongs to $\mathbf{Q}^{\prime}\left(\mathbb{R}^{s}\right)$ if $\mathbf{h}_{0}(z)=A(z) \mathbf{h}$.

For a data sequence $\left\{\mathbf{d}_{k} \in \mathbb{R}^{s}, k \in \mathbb{Z}\right\}$, the vector subdivision operator $S_{P}$ acting on it is defined by

$$
\left(S_{P} d\right)_{k}^{T}=\sum_{j \in \mathbb{Z}} \mathbf{d}_{j}^{T} P_{k-m j}, \quad k \in \mathbb{Z} .
$$

Actually the vector subdivision scheme $\mathbf{S}_{P, \mathbf{h}_{0}, \Phi_{0}}$ is a recursive algorithm based on the vector subdivision operator

$$
\mathbf{d}_{\ell+1, k}^{T}=S_{P} \mathbf{d}_{\ell, k}^{T}=\sum_{j \in \mathbb{Z}} \mathbf{d}_{\ell, j}^{T} P_{k-m j}, \quad k \in \mathbb{Z}, \ell \in \mathbb{N}
$$

and a convolution operator

$$
f_{\ell+1, k}=\sum_{j \in \mathbb{Z}} \mathbf{d}_{\ell+1, j}^{T} \Phi_{0}(k-j), \quad k \in \mathbb{Z}, \ell \in \mathbb{N}
$$

with the initial data $\mathbf{d}_{0}=\left\{\mathbf{d}_{0, k} \in \mathbb{R}^{s}: k \in \mathbb{Z}\right\}$ and the function

$$
\Phi_{0}(x)=\sum_{j \in \mathbb{S}} \mathbf{y}_{j} \mathscr{X}(x-j)
$$

where $\mathscr{X}(x)$ is the characteristic function of interval $[0,1)$ and $\mathbf{y}=\left\{\mathbf{y}_{j} \in \mathbb{R}^{s}, j \in \mathbb{S}\right\}$ is selected such that $\mathbf{y}(z) \in \mathbb{R}^{\prime}\left(\mathbb{R}^{s}\right)$ and $\mathbf{h}_{0}(z)^{T} \mathbf{y}(z)=1$.

Lemma 1. If $\mathbf{h}_{0}(z) \in \mathfrak{Q}\left(\mathbb{R}^{s}\right)$, then there exist linearly independent vectors $\mathbf{y}^{1}(z), \ldots, \mathbf{y}^{s}(z) \in \mathbf{Q}^{\prime}\left(\mathbb{R}^{s}\right)$ such that

$$
\mathbf{h}_{0}^{T}(z) \mathbf{y}^{j}(z)=1, \quad j=1, \ldots, s .
$$

Proof. Suppose that $\mathbf{h}_{0}^{T}=\mathbf{h}^{T}\left[\begin{array}{cc}1 & \sum_{j \in \mathbb{S}} \mathbf{r}_{j} z^{j} \\ \mathbf{0} & I_{s-1}\end{array}\right]$. It is not difficult to find that there exist linearly independent constant vectors $\mathbf{y}^{1}, \ldots, \mathbf{y}^{s}$ satisfying

$$
\mathbf{h}^{T} \mathbf{y}^{j}=1, \quad j=1, \ldots, s .
$$

Let

$$
\mathbf{y}^{j}(z)=\left[\begin{array}{cc}
1 & -\sum_{j \in \mathbb{S}} \mathbf{r}_{j} z^{j} \\
\mathbf{0} & I_{s-1}
\end{array}\right] \mathbf{y}^{j}, \quad j=1, \ldots, s .
$$

Then, by calculation the conclusion follows directly.

In order to define a sequence of continuous functions, we also need parameterization. As in [10] for the scalar case, we chose the associated parameter values:

$$
t_{k}^{\ell}:=t_{0}^{\ell}+\frac{k}{m^{\ell}}, \quad t_{0}^{\ell}=t_{0}^{\ell-1}-\frac{\tau}{m^{\ell}}, \quad t_{0}^{0}=0, k \in \mathbb{Z}, \ell \in \mathbb{N} .
$$

We call $\left\{t_{k}^{\ell}, k \in \mathbb{Z}, \ell \in \mathbb{N}\right\}$ the sequence of parameter values associated with the vector subdivision scheme. Let us define continuous functions $f_{\ell}$ by linearly interpolating the data $f_{\ell, k}$ to the parameter values $t_{k}^{\ell}$,

$$
f_{\ell}\left(t_{k}^{\ell}\right)=f_{\ell, k}, \quad k \in \mathbb{Z}, \quad \ell \in \mathbb{N} .
$$

Definition 2. If the sequence of continuous functions $\left\{f_{\ell}, \ell \in\right.$ $\mathbb{N}$ \} converges (in uniform norm), then we define the limit function as

$$
g_{\mathbf{d}_{0}}=\lim _{\ell \rightarrow \infty} f_{\ell} .
$$

We say that $g_{\mathbf{d}_{0}}$ is the limit function of the vector subdivision scheme $\mathbf{S}_{P, \mathbf{h}_{0}, \Phi_{0}}$ acting on the initial data $\mathbf{d}_{0}$. If $s=$ 1 and $\mathbf{h}_{0}=1$, one may find that the vector subdivision scheme is the same as the scalar one in [10]. The limit functions $\phi_{0}: \phi_{\boldsymbol{\delta}_{0}^{(p)}}:=g_{\boldsymbol{\delta}_{0}^{(p)}}, p=1, \ldots, s$, are called basic limit functions if the initial data is selected by

$$
\boldsymbol{\delta}_{0, k}^{(p)}=\left\{\begin{array}{ll}
\mathbf{e}_{p}, & k=0, \\
\mathbf{0}, & \text { otherwise, }
\end{array} \quad k \in \mathbb{Z}, p=1, \ldots, s\right.
$$

where $\mathbf{e}_{p}$ denotes $p$ th column of the $s \times s$ identity matrix. It is easy to check that $\Phi=\left(\phi_{1}, \ldots, \phi_{s}\right)^{T}$ is compactly supported and satisfies the refinement equation

$$
\Phi(t)=\sum_{k \in \mathbb{Z}} P_{k} \Phi(m t-k), \quad t \in \mathbb{R}
$$

In this paper, we consider vector subdivision scheme that is convergent and nonsingular, so that $g_{\mathbf{d}_{0}}=0$ if and only if $\mathbf{d}_{0}=\mathbf{0}$. Define the linear operator $Q_{P}$ (which is called cascade operator) as follows:

$$
Q_{P} \Phi=\sum_{k \in \mathbb{Z}} P_{k} \Phi(m \cdot-k), \quad \Phi \in\left(L^{2}(\mathbb{R})\right)^{s} .
$$


By induction on $n$, it is easy to verify that

$$
Q_{P}^{n} \Phi_{0}(x)=\sum_{k \in \mathbb{Z}} P_{n, k} \Phi_{0}\left(m^{n} x-k\right), \quad n \in \mathbb{N},
$$

where $\Phi_{0}$ is defined as (7),

$$
P_{n, k}=\sum_{j \in \mathbb{Z}} P_{n-1, j} P_{k-M j}, \quad P_{1, k}=P_{k}, \quad k \in \mathbb{Z} .
$$

Under the convergent condition of cascade algorithm (see [13]), we can prove that $Q_{P}^{n} \Phi_{0}$ converges to the normalized solution $\Phi$ of $(15)$ in $\left(L_{p}(\mathbb{R})\right)^{s}(1 \leq p \leq \infty)$.

Proposition 3. A vector subdivision scheme $\mathbf{S}_{P, \mathbf{h}_{0}, \Phi_{0}}$ is convergent and nonsingular if and only if (15) has compactly supported solution $\Phi$ and the integer translations of $\Phi$ are linearly independent.

Proof. Firstly, we claim that the convergence of vector subdivision scheme is the same as that of the cascade algorithm. Actually, by induction, for the initial data $\delta_{0}^{(p)}, p=1, \ldots, s$, we have

$$
\begin{aligned}
f_{1, j}^{(p)} & =\sum_{k \in \mathbb{Z}} \boldsymbol{\delta}_{1, k}^{(p)^{T}} \Phi_{0}(j-k) \\
& =\sum_{k \in \mathbb{Z}} \sum_{n \in \mathbb{Z}} \boldsymbol{\delta}_{0, n}^{(p)^{T}} P_{k-m n} \Phi_{0}(j-k) \\
& =\sum_{n \in \mathbb{Z}} \boldsymbol{\delta}_{0, n}^{(p)^{T}} \sum_{k \in \mathbb{Z}} P_{k} \Phi_{0}\left(m\left(m^{-1} j-n\right)-k\right) \\
& =\sum_{n \in \mathbb{Z}} \boldsymbol{\delta}_{0, n}^{(p)^{T}} Q_{P} \Phi_{0}\left(m^{-1} j-n\right) \\
& =\mathbf{e}_{p}^{T} Q_{P} \Phi_{0}\left(m^{-1} j\right),
\end{aligned}
$$

and

$$
f_{n, j}^{(p)}=\sum_{n \in \mathbb{Z}} \boldsymbol{\delta}_{0, n}^{(p)^{T}} Q_{P}^{n} \Phi_{0}(j-n)=\mathbf{e}_{p}^{T} Q_{P}^{n} \Phi_{0}\left(m^{-n} j\right) .
$$

Then, our declaration follows by a standard method in analysis. Note that the convergence of cascade algorithm with finite supported mask is equivalent to the existence of compactly supported solution of (15) (see [14]). We can obtain the desired result according to the proof of [11, Proposition 1.3].

Following $[10,11]$, we give the definitions of polynomial generation, polynomial reproduction, and stepwise polynomial reproduction with respect to vector subdivision scheme. We denote by $\Pi_{k}$ the space of polynomials up to degree $k$. Suppose $p_{\ell}=\left\{p_{\ell, k}, k \in \mathbb{Z}\right\}:=\left\{p\left(t_{k}^{\ell}\right), k \in \mathbb{Z}\right\}$, for $\ell \in \mathbb{N}$, and let $(\ell(\mathbb{Z}))^{s}$ be the space of $s$-vector sequence indexed by $\mathbb{Z}$.

Definition 4 (polynomial generation). A convergent stationary vector subdivision scheme $\boldsymbol{S}_{P, \mathbf{h}_{0}, \Phi_{0}}$ generates polynomials up to degree $n_{G}\left(\Pi_{n_{G}}\right.$-generating), if, for any polynomial $p(x) \in \Pi_{n_{G}}$, there exists some initial data $\mathbf{d}_{0} \in(\ell(\mathbb{Z}))^{s}$ such that $g_{\mathbf{d}_{0}}=p(x)$.
Definition 5 (polynomial reproduction). A convergent stationary vector subdivision scheme $\mathbf{S}_{P, \mathbf{h}_{0}, \Phi_{0}}$ with parameter values $t^{(\ell)}, \ell \in \mathbb{N}$ is reproducing polynomials up to degree $n_{R}\left(\Pi_{n_{R}}\right.$-reproducing), if, for the initial data $\mathbf{d}_{0}=\{(p *$ $\left.\left.\mathbf{h}_{0}\right)\left(t_{k}^{(0)}\right), k \in \mathbb{Z}\right\}$, with any polynomial $p(x) \in \Pi_{n_{R}}$, the limit of the vector subdivision scheme satisfies $g_{\mathbf{d}_{0}}=p(x)$.

Definition 6 (stepwise polynomial reproduction). A convergent stationary vector subdivision scheme $\mathbf{S}_{P, \mathbf{h}_{0}, \Phi_{0}}$ with parameter values $t^{(\ell)}, \ell \in \mathbb{N}$ is stepwise reproducing polynomials up to degree $n_{R}$ (stepwise $\Pi_{n_{R}}$-reproducing), if, for the data $\mathbf{d}_{\ell}=\left\{\left(p_{\ell} * \mathbf{h}_{0}\right)(k), k \in \mathbb{Z}\right\}$, with any polynomial $p(x) \in \Pi_{n_{R}}$,

$$
\begin{gathered}
\mathbf{d}_{\ell+1}^{T}=\left(\mathbf{S}_{P} \mathbf{d}^{T}\right)_{\ell} \quad \text { or, equivalently, } \\
\left(p_{\ell+1} * \mathbf{h}_{0}^{T}\right)(k)=\sum_{j \in \mathbb{Z}} \mathbf{d}_{\ell}^{T}\left(t_{j}^{(\ell)}\right) P_{k-m j}, \quad k \in \mathbb{Z} .
\end{gathered}
$$

Noting basic limit function $\Phi$ defined by (14), it is easy to show that

$$
g_{\mathbf{d}_{0}}=\sum_{k \in \mathbb{Z}} \mathbf{d}_{0, k}^{T} \Phi(\cdot-k) .
$$

Thus the equivalence of polynomial generation and accuracy (approximation order, sum rulers) is clear; see [6, 7]. The following proposition shows that for a nonsingular convergent vector subdivision scheme the concepts of polynomial reproduction and stepwise polynomial reproduction are equivalent.

Proposition 7. A nonsingular convergent vector subdivision scheme is stepwise $\Pi_{n_{R}}$ reproducing if and only if it is $\Pi_{n_{R}}$ reproducing.

Proof. Since that $\mathbf{h}_{0}^{T}(z) \mathbf{y}(z)=1$, then by discrete Fourier transform we have $\sum_{u \in \mathbb{S}} \mathbf{h}_{0, n-u}^{T} \mathbf{y}_{u}=\delta_{n}$. Thus under the stepwise polynomial reproduction, from (6) we can easily get, for $p(x) \in \Pi_{n_{R}}$,

$$
\begin{aligned}
f_{\ell, k} & =\sum_{j \in \mathbb{S}} \mathbf{d}_{\ell, k-j}^{T} \Phi_{0}(j) \\
& =\sum_{j \in \mathbb{S}}\left(p_{\ell} * \mathbf{h}_{0}^{T}\right)(k-j) \mathbf{y}_{j} \\
& =\sum_{j \in \mathbb{S}} p\left(t_{k-j}^{\ell}\right) \sum_{u \in \mathbb{S}} \mathbf{h}_{0, u}^{T} \mathbf{y}_{j-u}=p\left(t_{k}^{\ell}\right), \quad k \in \mathbb{Z}, \ell \in \mathbb{N} .
\end{aligned}
$$

As a result, $f_{\ell, k}=p\left(t_{k}^{\ell}\right)$ implies $f_{\ell+1, k}=p\left(t_{k}^{\ell+1}\right)$. The rest of necessary part one may refer to [11, Proposition 1.7]. For the sufficient part, noting (6) and (7), we have

$$
f_{\ell, k}=\sum_{j \in \mathbb{S}} \mathbf{d}_{\ell, k-j}^{T} \mathbf{y}_{j}, \quad k \in \mathbb{Z}, \quad \ell \in \mathbb{N} .
$$

Consider $p(x) \in \Pi_{n_{R}}$. Taking $f_{\ell, k}=p\left(t_{k}^{\ell}\right)$ and using the same methods of [11, Proposition 2.1], one obtains from (6) that

$$
f_{\ell+1, k}=p\left(t_{k}^{\ell+1}\right) \text {. }
$$


Noting that $\mathbf{h}_{0}(z)^{T} \mathbf{y}(z)=1$, we deduce from $f_{\ell, k}=p\left(t_{k}^{\ell}\right)$ that

$$
\begin{aligned}
0 & =\sum_{j \in \mathbb{S}} \mathbf{d}_{\ell, k-j}^{T} \mathbf{y}_{j}-\sum_{n} p\left(t_{k-n}^{\ell}\right) \delta_{n} \\
& =\sum_{j \in \mathbb{S}} \mathbf{d}_{\ell, k-j}^{T} \mathbf{y}_{j}-\sum_{n} p\left(t_{k-n}^{\ell}\right) \sum_{j \in \mathbb{S}} \mathbf{h}_{0, n-j}^{T} \mathbf{y}_{j} \\
& =\sum_{j \in \mathbb{S}}\left(\mathbf{d}_{\ell, k-j}^{T}-\left(p_{\ell} * \mathbf{h}_{0}^{T}\right)(k-j)\right) \mathbf{y}_{j} \triangleq \sum_{j \in \mathbb{S}} \mathbf{x}_{\ell, k-j} \mathbf{y}_{j},
\end{aligned}
$$

where $\mathbf{x}_{\ell, k}=\left[x_{k, 1}^{\ell}, x_{k, 2}^{\ell}, \ldots, x_{k, s}^{\ell}\right]$. Similarly, the equation $f_{\ell+1, k}=p\left(t_{k}^{\ell+1}\right)$ implies

$$
\sum_{j \in \mathbb{S}}\left(\mathbf{d}_{\ell+1, k-j}^{T}-\left(p_{\ell+1} * \mathbf{h}_{0}^{T}\right)(k-j)\right) \mathbf{y}_{j}=0, \quad k \in \mathbb{Z} .
$$

Following Lemma 1, we select linearly independent vectors $\mathbf{y}^{t}(z)$ as (10) satisfying $\mathbf{h}_{0}(z)^{T} \mathbf{y}^{t}(z)=1, t=1, \ldots, s$. Then,

$$
\begin{aligned}
& {[0, \ldots, 0]} \\
& \quad=\sum_{j \in \mathbb{S}} \mathbf{x}_{\ell, k-j}\left[\mathbf{y}_{j}^{1}, \ldots, \mathbf{y}_{j}^{s}\right] \\
& \quad=\sum_{j \in \mathbb{S} \backslash\{0\}} \mathbf{x}_{\ell, k-j}\left[\begin{array}{cc}
0 & \mathbf{r}_{j} \\
\mathbf{0} & O_{s-1}
\end{array}\right] Y+\left[x_{k, 1}^{\ell}, \ldots, x_{k, s}^{\ell}\right]\left[\begin{array}{cc}
1 & \mathbf{r}_{0} \\
\mathbf{0} & I_{s-1}
\end{array}\right] Y \\
& \quad=\left[x_{k, 1}^{\ell}, x_{k, 2}^{\ell}, \ldots, x_{k, s}^{\ell}\right] Y+\left[0, \sum_{j \in \mathbb{S}} x_{k-j, 1}^{\ell} \mathbf{r}_{j}\right] Y, \quad k \in \mathbb{Z},
\end{aligned}
$$

where $Y=\left[\mathbf{y}^{1}, \ldots, \mathbf{y}^{s}\right]$. Since $Y$ is an invertible matrix, then $f_{\ell, k}=p\left(t_{k}^{\ell}\right)$ is equivalent to $x_{k, 1}^{\ell}=x_{k, 2}^{\ell}=\cdots=x_{k, s}^{\ell}=0$. The conclusion follows directly.

\section{Algebraic Condition for Polynomial Reproduction}

In this section we firstly consider the nonsingular subdivision scheme with standard subdivision symbol $P(z)$ in (33) and provide algebraic conditions on $P(z)$ for checking the polynomial reproduction. With the two-scale similarity transform (TST) in the hand, we further discuss the properties of polynomial reproduction with respect to the general subdivision symbol satisfying certain order of sum rules. For TST, see $[6,13,15,16]$ and references therein.

We denote the subsymbols of a vector subdivision symbol $P(z)$ by

$$
P^{\alpha}(z)=\sum_{k \in \mathbb{Z}} P_{m k+\alpha} z^{m k+\alpha}, \quad \alpha=0, \ldots, m-1, z \in \mathbb{C} \backslash\{0\},
$$

and remark that the $j$ th derivative of a subsymbol is

$$
D^{(j)} P^{\alpha}(z)=\sum_{k \in \mathbb{Z}} q_{j}(m k+\alpha) z^{m k+\alpha-j},
$$

where $q_{j} \in \pi_{j}$ is the polynomial

$$
q_{j}(x)=\prod_{u=0}^{j-1}(x-u) .
$$

We further assume that

$$
P(z)=\left[\begin{array}{ll}
P_{11}(z) & P_{12}(z) \\
P_{21}(z) & P_{22}(z)
\end{array}\right]
$$

where $P_{11}(z), P_{12}(z), P_{21}(z)$, and $P_{22}(z)$ are $1 \times 1,1 \times(s-1)$, $(s-1) \times 1$, and $(s-1) \times(s-1)$ matrices of Laurent polynomials, respectively. The subsymbols of $P_{11}(z),\left(P_{12}(z)\right)$ are defined by $P_{11}^{\alpha}(z),\left(P_{12}^{\alpha}(z)\right), \alpha=0, \ldots, m-1$. We define standard subvision symbol with sum rules of order $n$ by

$$
P(z)=\left[\begin{array}{cc}
\left(1+z+\cdots+z^{m-1}\right)^{n} Q_{11}(z) & \left(1-z^{m}\right)^{n} Q_{12}(z) \\
P_{21}(z) & P_{22}(z)
\end{array}\right],
$$

where $Q_{11}(1)=1 / m^{n-1}$ and $Q_{11}(z), Q_{12}(z)$ are $1 \times 1,1 \times$ $(s-1)$ matrices, respectively. Similar to [10, Lemma 2.1] or [11, Proposition 2.1], we have the following proposition.

Proposition 8. The following are equivalent.

(i) The vector subdivision symbol $P(z)$ takes the form of (33).

(ii) The entries of $P(z)$ satisfy

$$
\begin{array}{r}
P_{11}(1)=m, \quad D^{(j)} P_{11}(\epsilon)=0, \quad j=0, \ldots, n-1, \\
\epsilon \in E^{\prime} ;
\end{array}
$$

$$
D^{(j)} P_{12}(\epsilon)=\mathbf{0}, \quad j=0, \ldots, n-1, \epsilon \in E,
$$

with $\mathbf{0} \in \mathbb{R}^{s-1}$, where $E=\left\{e^{-i(2 \pi k / m)}, k=0, \ldots, m-1\right\}$ and $E^{\prime}=E \backslash\{1\}$.

(iii) The subsymbols of the entries of $P(z)$ satisfy

$$
\begin{array}{r}
m D^{(j)} P_{11}^{\alpha}(1)=D^{(j)} P_{11}(1), \quad j=0, \ldots, n-1, \\
\alpha=0, \ldots, m-1 ;
\end{array}
$$

$D^{(j)} P_{12}^{\alpha}(1)=\mathbf{0}, \quad j=0, \ldots, n-1, \alpha=0, \ldots, m-1$.

(iv) The coefficients of the entries of $P(z)$ satisfy

$$
\begin{gathered}
m \sum_{k \in \mathbb{Z}} q_{j}(\ell-m k) P_{11, \alpha-m k}=D^{(j)} P_{11}(1), \\
\sum_{k \in \mathbb{Z}} q_{j}(\ell-m k) P_{12, \alpha-m k}=\mathbf{0}, \quad \ell \in \mathbb{Z}, \\
j=0, \ldots, n-1, \quad \alpha=0, \ldots, m-1 .
\end{gathered}
$$

Similar to [10, Lemma 4.2] or [11, Proposition 2.5], we also have the following proposition. It allows us to express the polynomial reproduction of $\mathbf{S}_{P, \mathbf{h}_{0}, \Phi_{0}}$ in terms of the properties of its symbol, where $P(z)$ takes the form of (33). 
Proposition 9. Let $n \in \mathbb{N}, \tau \in \mathbb{R}$, and $q_{j}$ as in (31). A vector subdivision symbol $P(z)$ satisfies

$$
\begin{gathered}
D^{(j)} P_{11}(1)=m q_{j}(\tau), \quad D^{(j)} P_{11}(\epsilon)=0, \quad j=0, \ldots, n, \\
\epsilon \in E^{\prime}, \\
D^{(j)} P_{12}(\epsilon)=\mathbf{0}, \quad j=0, \ldots, n, \epsilon \in E,
\end{gathered}
$$

if and only if

$$
\begin{gathered}
\sum_{k \in \mathbb{Z}} P_{11, u-m k} k^{j}=\left(\frac{u-\tau}{m}\right)^{j}, \quad u \in \mathbb{Z}, j=0, \ldots, n, \\
\sum_{k \in \mathbb{Z}} P_{12, u-m k} k^{j}=\mathbf{0}, \quad u \in \mathbb{Z}, j=0, \ldots, n .
\end{gathered}
$$

3.1. Standard Subdivision Symbol. Suppose that subdivision symbol has the standard form (33) in this subsection. Then the constant vector $\mathbf{h}_{0}$ and initial function $\Phi_{0}(x)$ in $\mathbf{S}_{P, \mathbf{h}_{0}, \Phi_{0}}$ can be chosen by

$$
\mathbf{h}_{0}=\mathbf{e}_{1}, \quad \Phi_{0}(x)=\mathbf{y} \mathscr{X}(x),
$$

respectively, where $\mathbf{y}$ satisfies $\mathbf{h}_{0}^{T} \mathbf{y}=1$. We provide a simple algebraic condition for determining $\tau \in \mathbb{R}$, which appears in (11) and guarantees the reproduction of linear polynomial. In Theorem 11, we then provide algebraic conditions on $P(z)$ for checking the reproduction of polynomials of higher degree.

Proposition 10. Let $\mathbf{h}_{0}, \Phi_{0}$ be defined by (39) and let $P$ take the form of (33) with $n=2$. Let $\mathbf{S}_{P, \mathbf{h}_{0}, \Phi_{0}}$ be a nonsingular vector subdivision scheme. Then, $\mathbf{S}_{P, \mathbf{h}_{0}, \Phi_{0}}$ reproduces linear polynomials if and only if its parameter values are given by (11) with

$$
\tau=\frac{D P_{11}(1)}{m}
$$

Proof. We follow the lines of Theorem 3.1 of [10] or Proposition 2.3 of [11]. According to Proposition 7, polynomial reproduction is equivalent to stepwise polynomial reproduction. Hence it is sufficient to show that $\mathbf{d}_{\ell, k}=\mathbf{h}_{0} p\left(t_{k}^{\ell}\right)$ implies $\mathbf{d}_{\ell+1, k}=\mathbf{h}_{0} p\left(t_{k}^{\ell+1}\right), k \in \mathbb{Z}$, for any $\ell \in \mathbb{N}$. Note that any convergent vector subdivision scheme reproduces constants; we only consider the monomial $p(x)=x$. Let $\ell \in \mathbb{N}$ and $\mathbf{d}_{\ell, k}=\mathbf{h}_{0} p\left(t_{k}^{\ell}\right)=\mathbf{h}_{0} \cdot\left(t_{0}^{\ell}+\left(j / m^{\ell}\right)\right), j \in \mathbb{Z}$. For any $k \in \mathbb{Z}$ and $\alpha=0, \ldots, m-1$, we have

$$
\begin{aligned}
\mathbf{d}_{\ell+1, m k+\alpha}^{T} & =\sum_{j} \mathbf{d}_{\ell, j}^{T} P_{m k+\alpha-m j} \\
& =\sum_{j} \mathbf{d}_{\ell, k-j}^{T} P_{m j+\alpha} \\
& =\sum_{j}\left(\mathbf{h}_{0}^{T}\left(t_{0}^{\ell}+\frac{k-j}{m^{\ell}}\right)\right) P_{m j+\alpha}
\end{aligned}
$$

$$
\begin{gathered}
=\sum_{j}\left(\mathbf{h}_{0}^{T}\left(t_{0}^{\ell}+\frac{m k+\alpha}{m^{\ell+1}}\right)\right) P_{m j+\alpha} \\
-\sum_{j}\left(\mathbf{h}_{0}^{T}\left(t_{0}^{\ell}+\frac{m j+\alpha}{m^{\ell+1}}\right)\right) P_{m j+\alpha} \\
=\left(\mathbf{h}_{0}^{T} t_{0}^{\ell}+\frac{m k+\alpha}{m^{\ell+1}}\right)-\frac{\mathbf{h}_{0}^{T} \mathbf{D} P(1)}{m^{\ell+1}}, \\
\mathbf{d}_{\ell+1, m k+\alpha}^{T}=\mathbf{h}_{0}^{T} t_{0}^{\ell}-\frac{\mathbf{h}_{0}^{T} \tau}{m^{\ell+1}}+\mathbf{h}_{0}^{T} \frac{m k+\alpha}{m^{\ell+1}} .
\end{gathered}
$$

As a result, the vector subdivision scheme reproduces linear polynomials stepwisely if and only if

$$
\mathbf{h}_{0}^{T} \tau=\mathbf{h}_{0}^{T} D P(1) .
$$

Then, (40) follows directly.

Theorem 11. Let $n_{R} \in \mathbb{N}_{0}$. A convergent subdivision scheme with symbol $P(z)$ in (33) and associated parameterization in (11), for some $\tau \in \mathbb{R}$, reproduces polynomials of degree up to $n_{R}$ if and only if

$$
\begin{gathered}
D^{(k)} P_{11}(1)=m q_{k}(\tau), \quad D^{(k)} P_{11}(\epsilon)=0, \quad k=0, \ldots, n_{R}, \\
\epsilon \in E^{\prime}, \\
D^{(k)} P_{12}(\epsilon)=\mathbf{0}, \quad k=0, \ldots, n_{R}, \epsilon \in E .
\end{gathered}
$$

Proof. The proof is induction. In the case $k=0$ the conclusion follows by Proposition 8. By Proposition 7, it suffices to prove the results for the step polynomial reproduction.

" $\Leftarrow$ :" Let $k \leq n_{R}$ and $\ell>0$. Suppose that $p(x)=c_{k} x^{k}+$ $\widetilde{p}(x)$, with $\tilde{p} \in \Pi_{k-1}$. We show that

$$
\mathbf{d}_{\ell}=\left\{\mathbf{h}_{0} p\left(t_{j}^{\ell}\right)=\mathbf{h}_{0} c_{k}\left(t_{j}^{\ell}\right)^{k}+\mathbf{h}_{0} \tilde{p}\left(t_{j}^{\ell}\right), j \in \mathbb{Z}\right\}
$$

satisfies $\mathbf{d}_{\ell+1}^{T}=S_{P} \mathbf{d}_{\ell}^{T}=\left\{\mathbf{h}_{0}^{T} p\left(t_{j}^{\ell+1}\right), j \in \mathbb{Z}\right\}$. Actually by induction and stepwise polynomial reproducing, we have

$$
\begin{aligned}
\mathbf{d}_{\ell+1, j}^{T} & =\sum_{u \in \mathbb{Z}} \mathbf{d}_{\ell, u}^{T} P_{j-m u} \\
& =\sum_{u \in \mathbb{Z}} \mathbf{h}_{0}^{T} c_{k}\left(t_{0}^{\ell}+\frac{u}{m^{\ell}}\right)^{k} P_{j-m u}+\widetilde{p}\left(t_{j}^{\ell+1}\right) \\
& =\sum_{u \in \mathbb{Z}} \mathbf{h}_{0}^{T} c_{k} \sum_{p=0}^{k}\left(\begin{array}{l}
k \\
p
\end{array}\right)\left(\frac{u}{m^{\ell}}\right)^{p}\left(t_{0}^{\ell}\right)^{k-p} P_{j-m u}+\tilde{p}\left(t_{j}^{\ell+1}\right) \\
& =\mathbf{h}_{0}^{T} c_{k} \sum_{p=0}^{k}\left(t_{0}^{\ell}\right)^{k-p} \sum_{u \in \mathbb{Z}}\left(\begin{array}{l}
k \\
p
\end{array}\right)\left(\frac{u}{m^{\ell}}\right)^{p} P_{j-m u}+\tilde{p}\left(t_{j}^{\ell+1}\right) .
\end{aligned}
$$


By Proposition 9, (43) is equivalent to

$$
\begin{aligned}
\mathbf{h}_{0}^{T} \sum_{u \in \mathbb{Z}} u^{p} P_{j-m u} & =\sum_{u \in \mathbb{Z}} u^{p}\left[P_{11, j-m u}, P_{12, j-m u}\right] \\
& =\mathbf{h}_{0}^{T}\left(\frac{j-\tau}{m}\right)^{p} .
\end{aligned}
$$

Then,

$$
\begin{aligned}
\mathbf{d}_{\ell+1, j}^{T} & =\mathbf{h}_{0}^{T} c_{k} \sum_{p=0}^{k}\left(\begin{array}{l}
k \\
p
\end{array}\right)\left(t_{0}^{\ell}\right)^{k-p}\left(\frac{j-\tau}{m^{\ell+1}}\right)^{p}+\mathbf{h}_{0}^{T} \tilde{p}\left(t_{j}^{\ell+1}\right) \\
& =\mathbf{h}_{0}^{T} c_{k}\left(t_{0}^{\ell}+\frac{j-\tau}{m^{\ell+1}}\right)^{k}+\mathbf{h}_{0}^{T} \tilde{p}\left(t_{j}^{\ell+1}\right) \\
& =\mathbf{h}_{0}^{T} c_{k}\left(t_{j}^{\ell+1}\right)^{k}+\mathbf{h}_{0}^{T} \tilde{p}\left(t_{j}^{\ell+1}\right) .
\end{aligned}
$$

“ $\Rightarrow:$ " Assume that the vector subdivision scheme is $\Pi_{n_{R}}$ reproducing. Let $p(x)=x^{k}\left(k \leq n_{R}\right)$ and $\mathbf{d}_{\ell}=\left\{\mathbf{h}_{0} p\left(t_{j}^{\ell}\right), j \in\right.$ $\mathbb{Z}$ \}, with $\mathbf{h}_{0}=\mathbf{e}_{1}$. Using the same method as above, we have

$$
\mathbf{d}_{\ell+1, j}^{T}=\mathbf{h}_{0}^{T} \sum_{p=0}^{k}\left(\begin{array}{l}
k \\
p
\end{array}\right)\left(t_{0}^{\ell}\right)^{k-p} \sum_{u \in \mathbb{Z}}\left(\frac{u}{m^{\ell}}\right)^{p} P_{j-m u} .
$$

On the other hand,

$$
p\left(t_{j}^{\ell+1}\right)=\sum_{p=0}^{k}\left(\begin{array}{l}
k \\
p
\end{array}\right)\left(t_{0}^{\ell}\right)^{k-p}\left(\frac{j-\tau}{m^{\ell+1}}\right)^{p} .
$$

Combining the above two equations, we deduce

$\sum_{p=0}^{k}\left(\begin{array}{l}k \\ p\end{array}\right)\left(t_{0}^{\ell}\right)^{k-p}\left(\frac{1}{m^{\ell}}\right)^{p}\left[\sum_{u \in \mathbb{Z}} u^{p} \mathbf{h}_{0}^{T} P_{j-m u}-\mathbf{h}_{0}^{T}\left(\frac{j-\tau}{m}\right)^{p}\right]=0$.

By induction, we obtain

$$
\sum_{u \in \mathbb{Z}} u^{k} \mathbf{h}_{0}^{T} P_{j-m u}-\mathbf{h}_{0}^{T}\left(\frac{j-\tau}{m}\right)^{k}=0 .
$$

As a result, by Proposition 9 the conclusion (43) follows.

3.2. General Subdivision Symbol. We devote this section to discussing the polynomial reproduction of vector subdivision scheme with general subdivision symbol. Suppose that subdivision symbol $P(z)$ has sum rules of order $n$ (see $[6,7]$ ), that is, if there exists a row vector $\mathbf{y}(\xi)$ of trigonometric polynomials such that $\mathbf{y}(0) \neq 0$ and

$$
\begin{array}{r}
{[\mathbf{y}(d \cdot) P(\cdot)]^{(j)}\left(\frac{2 \pi k}{m}\right)=\delta_{k} \mathbf{y}^{(j)}(0), \quad j=0, \ldots, n-1 ;} \\
k=0, \ldots, m-1 .
\end{array}
$$

In [16, Theorem 2.2], they showed that subdivision symbol $\widetilde{P}(\xi)$ satisfies the sum rules of order $n$, if and only if there exists an invertible $s \times s$ matrix

$$
U(z)=\left[\begin{array}{cc}
1 & \sum_{j \in S} \mathbf{r}_{j} z^{j} \\
\mathbf{0} & I_{s-1}
\end{array}\right] U^{0}
$$

such that $\widetilde{P}(z)=U\left(z^{m}\right) P(z) U^{-1}(z)$ with $P(z)$ taking the form (33), where $U^{0}$ is a constant invertible matrix. It is evident that

$$
U^{-1}(z)=V(z):=V^{0}\left[\begin{array}{cc}
1 & -\sum_{j \in S} \mathbf{r}_{j} z^{j} \\
\mathbf{0} & I_{s-1}
\end{array}\right]
$$

with $V^{0} U^{0}=I_{s}$.

Let the constant vector $\mathbf{h}_{0}$ and initial function $\Phi_{0}(x)$ in $\mathbf{S}_{P, \mathbf{h}_{0}, \Phi_{0}}$ be defined by (39). Then we choose $\widetilde{\mathbf{h}}_{0}$ and $\widetilde{\Phi}_{0}(x)$ in $\mathbf{S}_{\widetilde{P}, \widetilde{\mathbf{h}}_{0}, \widetilde{\Phi}_{0}}$ by

$$
\widetilde{\mathbf{h}}_{0}^{T}(z)=\mathbf{h}_{0}^{T} V(z), \quad \widetilde{\Phi}_{0}(x)=\sum_{j \in \mathbb{S}} \widetilde{\mathbf{y}}_{j} \mathscr{X}(x-j),
$$

with $\widetilde{\mathbf{y}}(z)=U(z) \mathbf{y}$. It is easy to see that $\widetilde{\mathbf{h}}_{0}(z) \in \mathfrak{Q}\left(\mathbb{R}^{s}\right), \widetilde{\mathbf{y}}(z) \in$ $\mathbf{Q}^{\prime}\left(\mathbb{R}^{s}\right)$, and $\widetilde{\mathbf{h}}_{0}^{T}(z) \widetilde{\mathbf{y}}(z)=1$.

Theorem 12. The vector subdivision scheme $\mathbf{S}_{\widetilde{P}, \widetilde{\mathbf{h}}_{0}, \widetilde{\Phi}_{0}}$ is $\Pi_{n_{R}}$ reproducing, if and only if $\mathbf{S}_{P, \mathbf{h}_{0}, \Phi_{0}}$ is $\Pi_{n_{R}}$ reproducing.

Proof. By Proposition 7, we only need to consider the equivalence under the condition of stepwise $\Pi_{n_{R}}$ reproducing. We just show the sufficient part (the necessary part can be proved similarly).

Suppose $p(x) \in \Pi_{n_{R}}$ and $\mathbf{d}_{\ell}=\left\{\mathbf{d}_{\ell, k}:=\mathbf{h}_{0} p\left(t_{k}^{(\ell)}\right), k \in \mathbb{Z}\right\}$, where $\ell \in \mathbb{N}$ and parameter values $t_{k}^{(\ell)}$ are defined by (11). By the definition of stepwise polynomial reproduction, we have

$$
\mathbf{d}_{\ell+1, k}=\sum_{j \in \mathbb{Z}} \mathbf{d}_{\ell}^{T}\left(t_{j}^{(\ell)}\right) P_{k-m j}, \quad k \in \mathbb{Z} .
$$

Since $V(z) U(z)=1$, then we get $\sum_{u \in \mathbb{S}} V_{u} U_{j-u}=\delta_{j} I_{s}$, where $U_{j}, j \in \mathbb{Z}$ are the coefficients of $U(z)$ with $U_{j}=O_{s}, j \notin$ $\mathbb{S}$ and $V_{j}, j \in \mathbb{Z}$ are defined similarly. For the new scheme $\mathbf{S}_{\widetilde{P}, \widetilde{\mathbf{h}}_{0}, \widetilde{\Phi}}$, we have

$$
\widetilde{\mathbf{d}}_{\ell, j}^{T}=\left(\mathbf{p}_{\ell} * \widetilde{\mathbf{h}}_{0}^{T}\right)(j)=\sum_{q \in \mathbb{S}} \mathbf{h}_{0}^{T} p\left(t_{j-q}^{\ell}\right) V_{q} .
$$

Then

$$
\begin{aligned}
\left(\mathbf{S}_{\widetilde{P}} \tilde{d}_{\ell}^{T}\right)_{k} & =\sum_{j \in \mathbb{Z}} \widetilde{\mathbf{d}}_{\ell}^{T}\left(t_{j}^{(\ell)}\right) \widetilde{P}_{k-m j} \\
& =\sum_{j \in \mathbb{Z}} \widetilde{\mathbf{d}}_{\ell}^{T}\left(t_{j}^{(\ell)}\right) \sum_{v \in \mathbb{Z}} \sum_{u \in \mathbb{S}} U_{u} P_{v-m u} V_{k-m j-v} \\
& =\sum_{v \in \mathbb{Z}} \sum_{u \in \mathbb{S}} \sum_{j \in \mathbb{Z}} \sum_{q \in \mathbb{S}} \mathbf{h}_{0}^{T} p\left(t_{j-q}^{\ell}\right) V_{q} U_{u} P_{v-m u} V_{k-m j-v} \\
& =\sum_{v \in \mathbb{Z}} \sum_{u \in \mathbb{Z}} \sum_{j \in \mathbb{Z}} \mathbf{h}_{0}^{T} p\left(t_{j}^{\ell}\right) \sum_{q \in \mathbb{S}} V_{q} U_{u-j-q} P_{v-m u} V_{k-v} \\
& =\sum_{v \in \mathbb{Z}} \sum_{u \in \mathbb{Z}} \mathbf{h}_{0}^{T} p\left(t_{u}^{\ell}\right) P_{v-m u} V_{k-v} .
\end{aligned}
$$

Here, necessary variable substitution is used. Following the stepwise polynomial reproduction of $\mathbf{S}_{P \mathbf{h}_{0}, \Phi_{0}}$, we know that

$$
\left(\mathbf{S}_{\tilde{P}} \tilde{d}_{\ell}\right)_{k}=\sum_{v \in \mathbb{Z}} \mathbf{h}_{0}^{T} p\left(t_{v}^{\ell+1}\right) V_{k-v}=\tilde{\mathbf{d}}_{\ell+1}\left(t_{k}^{\ell+1}\right) .
$$

Thus the proof is completed. 


\section{Examples}

Example 1 (see [17, Example 4.2], GHM refinable mask). The vector subdivision scheme $\mathbf{S}_{\widetilde{P}, \widetilde{\mathbf{h}}_{0}, \widetilde{\Phi}_{0}}$ is based on the following mask symbol,

$$
\begin{aligned}
\widetilde{P}(z) & =\frac{1}{2} \sum_{k=0}^{3} P_{k} z^{k} \\
& =\frac{1}{40}\left[\begin{array}{cc}
12(1+z) & 16 \sqrt{2} \\
-\sqrt{2}(z+1)\left(z^{2}-10 z+1\right) & -6+20 z-6 z^{2}
\end{array}\right] .
\end{aligned}
$$

It is not difficult to find that the subdivision mask $\widetilde{P}$ satisfies the sum rules of order 2 . The corresponding vector $y(z)$ in (52) is selected by

$$
y(z)=\left[\frac{\sqrt{2}}{2}\left(1+z^{-1}\right) z^{-1}\right] .
$$

We construct a trigonometric matrix $U(z)$ by

$$
U(z)=\left[\begin{array}{cc}
\frac{\sqrt{2}}{2}(3-z) & 2-z \\
1 & \sqrt{2}
\end{array}\right]
$$

Then, $P(z)$ takes the form of (33),

$$
\left[\begin{array}{cc}
(1+z)^{2} Q_{11}(z) & \left(1-z^{2}\right)^{2} Q_{12}(z) \\
Q_{21}(z) & Q_{22}(z)
\end{array}\right]
$$

with

$$
\begin{gathered}
Q_{11}(z)=\frac{1}{10}\left(z^{3}-14 z^{2}+20 z-2\right) ; \\
Q_{12}(z)=\frac{\sqrt{2}}{20}\left(z^{2}-14 z+22\right) ; \\
Q_{21}(z)=-\frac{\sqrt{2}}{10}\left(z^{3}-12 z^{2}-5 z\right) ; \\
Q_{22}(z)=-\frac{1}{10}\left(z^{4}-14 z^{3}+22 z^{2}-5\right) .
\end{gathered}
$$

Using Proposition 10 we get that, for $\tau=0$, the scheme reproduces linear polynomials. Since $D^{(2)} P_{11}(1)=$ $-59 / 5 \neq 2 q_{2}(\tau)=0$, the scheme cannot reproduce polynomials of degree 2. For their graphs, see Figure 1.
Example 2 (see [17, Example 5]). The vector subdivision scheme $\mathbf{S}_{\widetilde{P}, \widetilde{\mathbf{h}}_{0}, \widetilde{\Phi}_{0}}$ is based on the mask symbol $\widetilde{P}(z)$ with

$$
\begin{gathered}
\widetilde{P}_{11}(z)=\frac{(10-3 \sqrt{10})}{80(1+z)\left(1+(38+12 \sqrt{10}) z+z^{2}\right)}, \\
\widetilde{P}_{12}(z)=\frac{(5 \sqrt{6}-2 \sqrt{15})}{80(1-z)(1+z)^{2}}, \\
\widetilde{P}_{21}(z)=\frac{(5 \sqrt{6}-3 \sqrt{15})}{80(1-z)\left(1-10(3+\sqrt{3}) z+z^{2}\right)}, \\
\widetilde{P}_{22}(z)=\frac{(5-3 \sqrt{10})}{1040(1+z)\left(13-(10+6 \sqrt{10}) z+13 z^{2}\right)} .
\end{gathered}
$$

The subdivision mask $\widetilde{P}$ satisfies the sum rules of order 3; see [17]. The corresponding vector $y(z)$ in (52) is selected by

$$
\begin{aligned}
y(z)= & {\left[\frac{-2+\sqrt{10}}{12}\left(\frac{1+\sqrt{10}}{z^{2}}+\frac{4+\sqrt{10}}{z}-1\right)\right.} \\
& \left.\times \frac{\sqrt{6}(-2+\sqrt{10})}{12}\left(z^{-2}-z^{-1}\right)\right] .
\end{aligned}
$$

If $U(z)$ is chosen by

$$
U(z)=\left[\begin{array}{cc}
1 & \frac{\sqrt{15}-\sqrt{6}}{12}(z-1)(z-3) \\
0 & 1
\end{array}\right]
$$

then $P(z)=U\left(z^{m}\right) \widetilde{P}(z) U^{-1}(z)$ takes the form of (33), with $n=3, m=2$. Using Proposition 10 we get that, for $\tau=3 / 2$, the scheme reproduces linear polynomials. Since $D^{(2)} P_{11}(1)=\sqrt{10}-2 \neq 2 q_{2}(\tau)=3 / 2$, the scheme cannot reproduce polynomials of degree 2 . For their graphs, see Figure 2.

Example 3 (see [16, Example 5.2]). The vector subdivision scheme $\mathbf{S}_{\widetilde{P}, \widetilde{\mathbf{h}}_{0}, \widetilde{\Phi}_{0}}$ is based on the following mask symbol:

$$
\widetilde{P}(z)=\left[\begin{array}{cc}
\frac{1}{2}+\frac{z}{4}+\frac{1}{4 z} & \frac{3}{8 z}-\frac{3 z}{8} \\
\frac{z}{16}-\frac{1}{16 z} & \frac{1}{4}-\frac{1}{16 z}-\frac{z}{16}
\end{array}\right]
$$

The subdivision mask $\widetilde{P}$ satisfies the sum rules of order 4 ; see [16] or [18]. If $U(z)$ is chosen by

$$
U(z)=\left[\begin{array}{cc}
1 & \frac{1}{12}\left(z^{2}-z^{-2}\right)-\frac{2}{3}\left(z-z^{-1}\right) \\
0 & 1
\end{array}\right]
$$




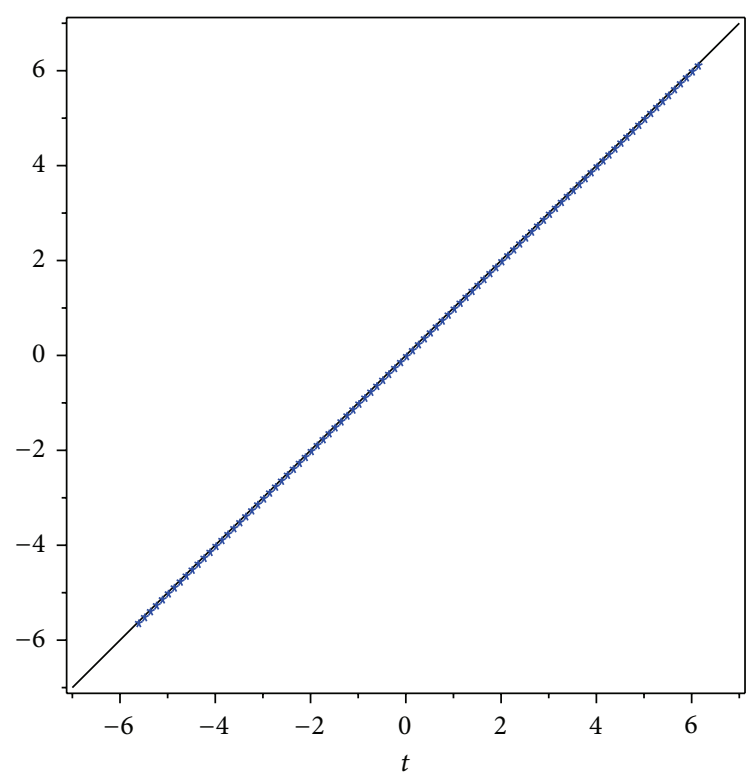

(a)

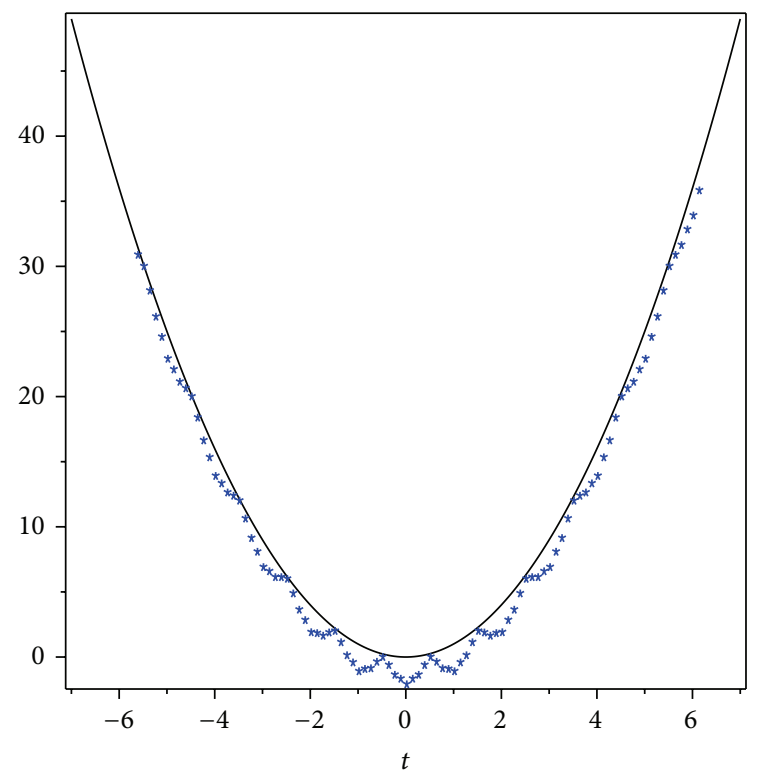

(b)

Figure 1: Polynomial reproduction of scheme $\mathbf{S}_{\widetilde{P}, \widetilde{\mathbf{h}}_{0}, \widetilde{\Phi}_{0}}$ with scheme mask symbol (60); solid line denotes the true polynomial and asterisk point denotes the discrete data using 5 times iteration of subdivision operator; (a) $p(t)=t$; (b) $p(t)=t^{2}$.

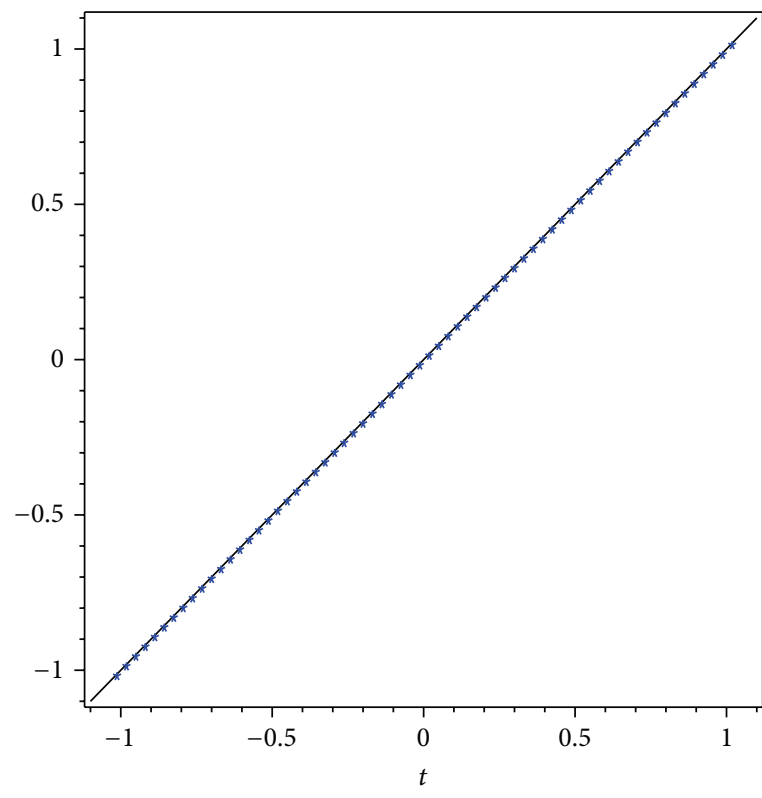

(a)

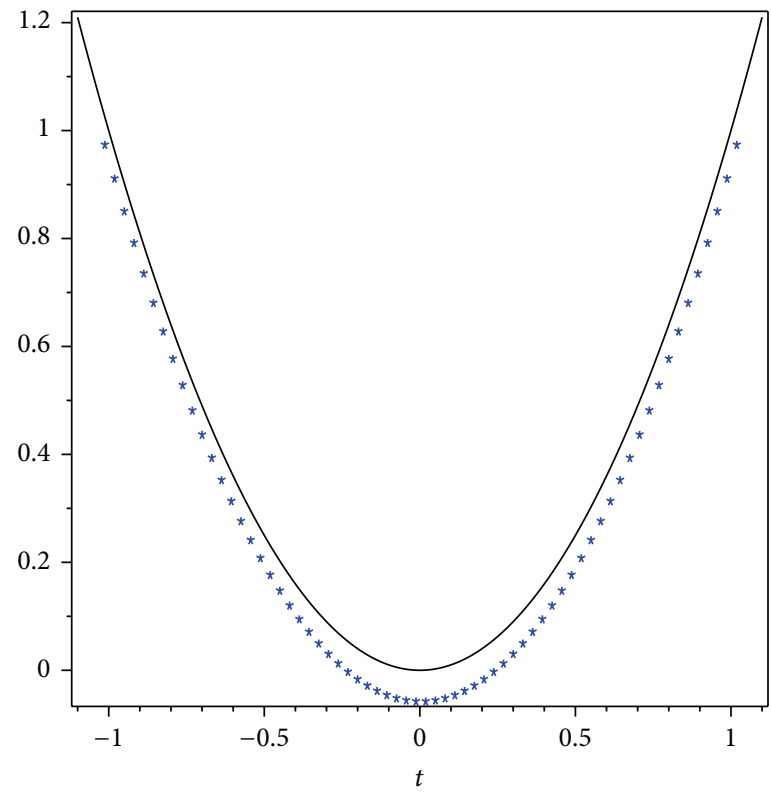

(b)

Figure 2: Polynomial reproduction of scheme $\mathbf{S}_{\widetilde{P}, \widetilde{\mathbf{h}}_{0}, \widetilde{\Phi}_{0}}$ with scheme mask symbol (65); solid line denotes the true polynomial and asterisk point denotes the discrete data using 5 times iteration of subdivision operator; (a) $p(t)=t$; (b) $p(t)=t^{2}$.

then $P(z)$ takes the form of (33),

$$
\left[\begin{array}{cc}
(1+z)^{4} Q_{11}(z) & \left(1-z^{2}\right)^{4} Q_{12}(z) \\
Q_{21}(z) & Q_{22}(z)
\end{array}\right]
$$

with

$$
\begin{aligned}
& Q_{11}(z)=\frac{1}{96 z^{5}}\left(z^{6}-4 z^{5}+z^{4}+16 z^{3}+z^{2}-4 z+1\right) \\
& Q_{12}(z)=\frac{1}{1152 z^{7}}\left(1-z^{2}\right)\left(z^{4}-8 z^{3}+8 z^{2}-8 z+1\right)
\end{aligned}
$$




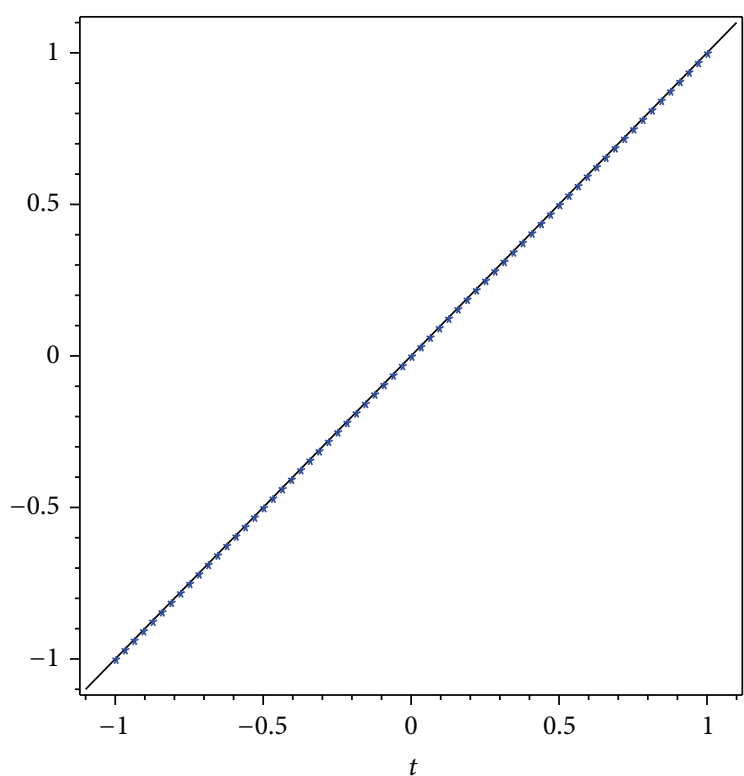

(a)

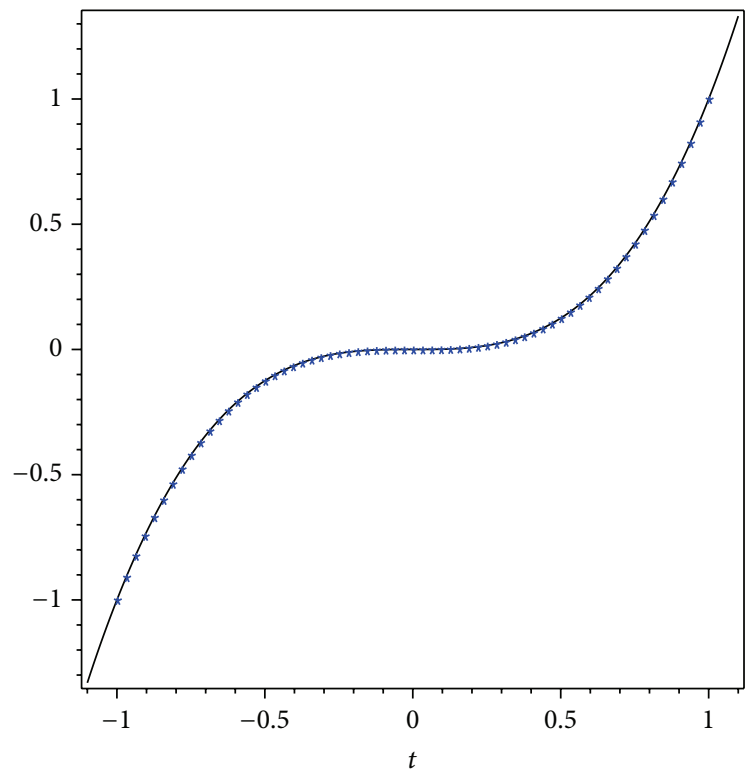

(c)

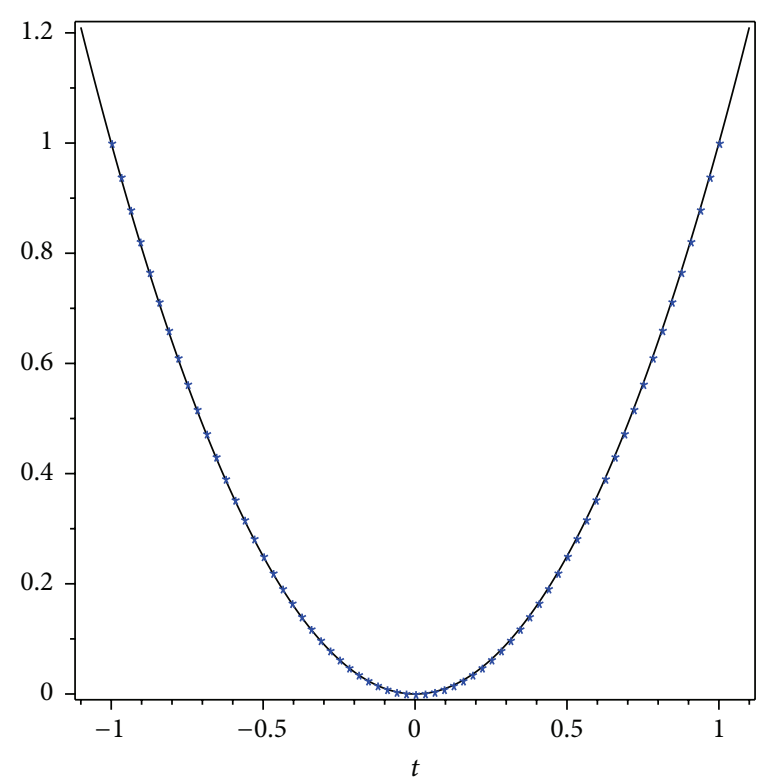

(b)

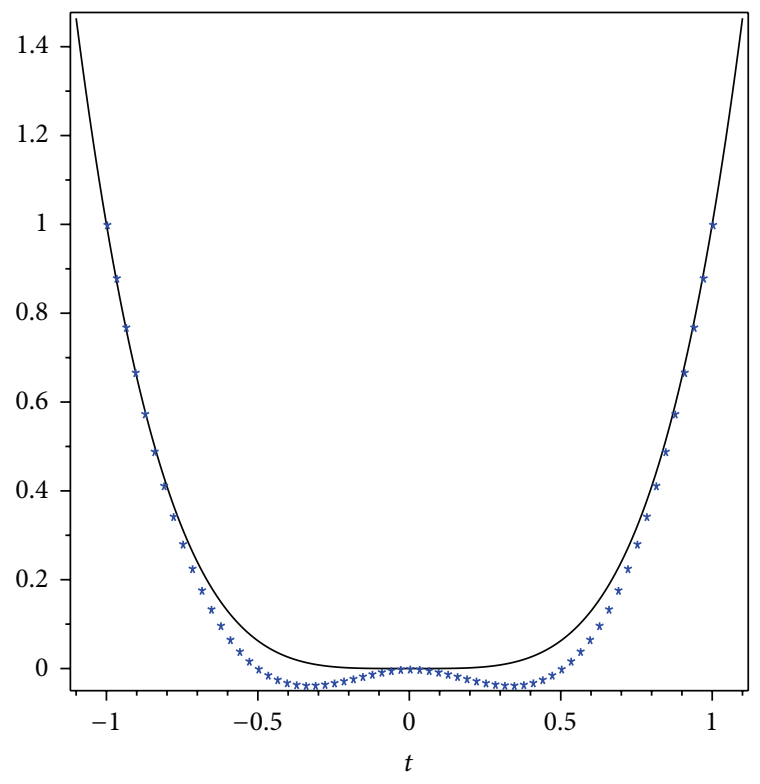

(d)

FIGURE 3: Polynomial reproduction of scheme $\mathbf{S}_{\widetilde{P}, \widetilde{\mathbf{h}}_{0}, \widetilde{\Phi}_{0}}$ with scheme mask symbol (68); solid line denotes the true polynomial and asterisk point denotes the discrete data using 5 times iteration of subdivision operator; (a) $p(t)=t$; (b) $p(t)=t^{2}$; (c) $p(t)=t^{3}$; (d) $p(t)=t^{4}$.

$$
\begin{gathered}
Q_{21}(z)=-\frac{\sqrt{1}}{8 z}(z-1)(z+1) ; \\
Q_{22}(z)=-\frac{1}{96 z^{3}}\left(z^{6}-8 z^{5}+11 z^{4}-32 z^{3}+11 z^{2}-8 z+1\right) .
\end{gathered}
$$

Using Proposition 10 we get that, for $\tau=0$, the scheme reproduces linear polynomials. Since $D^{(3)} P_{11}(1)=D^{(2)} P_{11}(1)=0$, the scheme also can reproduce polynomials up to degree 3 . For their graphs, see Figure 3.

\section{Conflict of Interests}

The authors declare that there is no conflict of interests regarding the publication of this paper.

\section{Acknowledgments}

This work was supported by the National Natural Science Foundation of China (Grant no. 11071152) and the Natural Science Foundation of Guangdong Province (Grant nos. 10151503101000025 and S2011010004511). 


\section{References}

[1] A. S. Cavaretta, W. Dahmen, and C. A. Micchelli, "Stationary subdivision," Memoirs of the American Mathematical Society, vol. 93, no. 453, 1991.

[2] N. Dyn and D. Levin, "Subdivision schemes in geometric modelling," Acta Numerica, vol. 11, pp. 73-144, 2002.

[3] C. Conti and G. Zimmermann, "Interpolatory rank-1 vector subdivision schemes," Computer Aided Geometric Design, vol. 21, no. 4, pp. 341-351, 2004.

[4] K. Jetter and G. Zimmermann, "Polynomial reproduction in subdivision," Advances in Computational Mathematics, vol. 20, no. 1-3, pp. 67-86, 2004.

[5] B. Han, "Vector cascade algorithms and refinable function vectors in Sobolev spaces," Journal of Approximation Theory, vol. 124, no. 1, pp. 44-88, 2003.

[6] F. Keinert, Wavelets and Multiwavelets, Chapman \& Hall/CRC, Boca Raton, Fla, USA, 2004.

[7] G. Plonka, "Approximation order provided by refinable function vectors," Constructive Approximation, vol. 13, no. 2, pp. 221244, 1997.

[8] K. Hormann and M. A. Sabin, "A family of subdivision schemes with cubic precision," Computer Aided Geometric Design, vol. 25, no. 1, pp. 41-52, 2008.

[9] N. Dyn, K. Hormann, M. A. Sabin, and Z. Shen, "Polynomial reproduction by symmetric subdivision schemes," Journal of Approximation Theory, vol. 155, no. 1, pp. 28-42, 2008.

[10] C. Conti and K. Hormann, "Polynomial reproduction for univariate subdivision schemes of any arity," Journal of Approximation Theory, vol. 163, no. 4, pp. 413-437, 2011.

[11] M. Charina and C. Conti, "Polynomial reproduction of multivariate scalar subdivision schemes," Journal of Computational and Applied Mathematics, vol. 240, pp. 51-61, 2013.

[12] M. Charina and L. Romani, "Polynomial reproduction of multivariate scalar subdivisionschemes with general dilation," http://arxiv.org/abs/1211.3264.

[13] S. Li, "Vector subdivision schemes in $\left(L_{p}\left(\mathbb{R}^{s}\right)\right)^{r}(1 \leq p \leq \infty)$ spaces," Science in China A: Mathematics, vol. 46, no. 3, pp. 364375, 2003.

[14] D.-R. Chen, R.-Q. Jia, and S. D. Riemenschneider, "Convergence of vector subdivision schemes in Sobolev spaces," Applied and Computational Harmonic Analysis, vol. 12, no. 1, pp. 128-149, 2002.

[15] V. Strela, Multiwavelets: theory and applications [Ph.D. thesis], Citeseer, 1996.

[16] B. Han and Q. Mo, "Multiwavelet frames from refinable function vectors," Advances in Computational Mathematics, vol. 18, no. 2-4, pp. 211-245, 2003.

[17] C. K. Chui and J.-A. Lian, "A study of orthonormal multiwavelets," Applied Numerical Mathematics, vol. 20, no. 3, pp. 273-298, 1996.

[18] B. Han, "Approximation properties and construction of Hermite interpolants and biorthogonal multiwavelets," Journal of Approximation Theory, vol. 110, no. 1, pp. 18-53, 2001. 


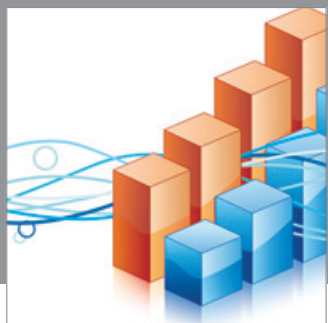

Advances in

Operations Research

mansans

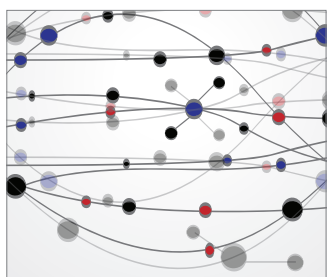

The Scientific World Journal
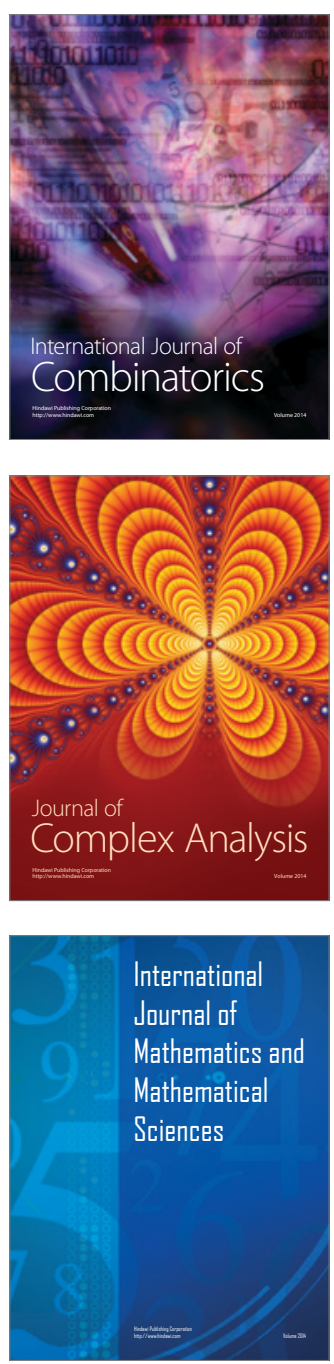
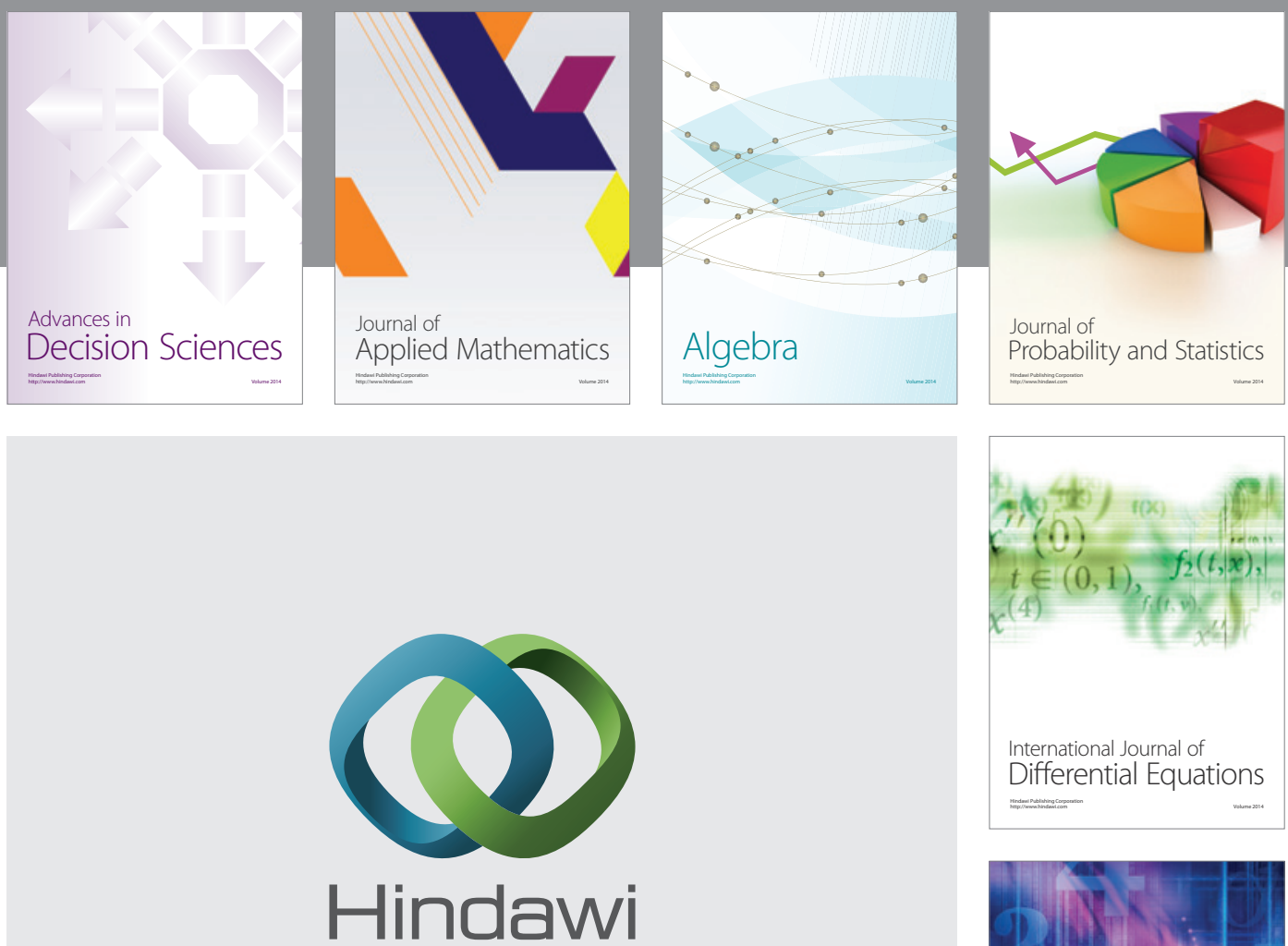

Submit your manuscripts at http://www.hindawi.com
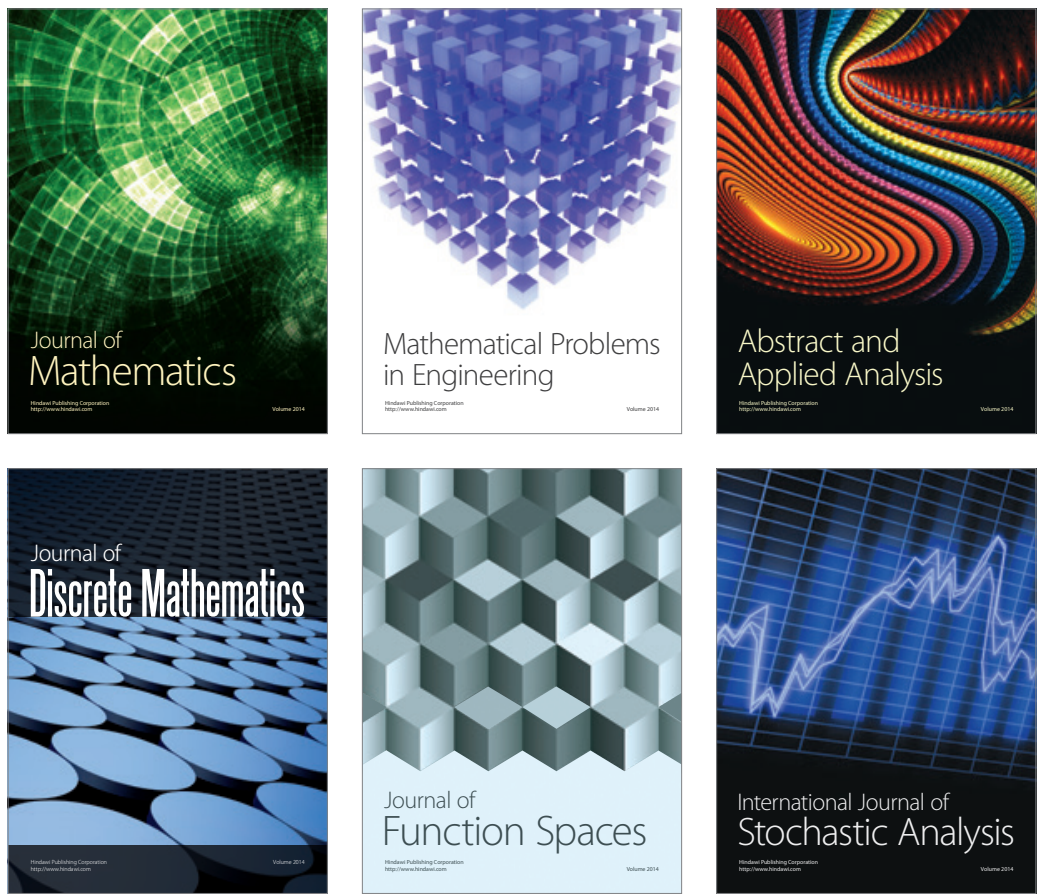

Journal of

Function Spaces

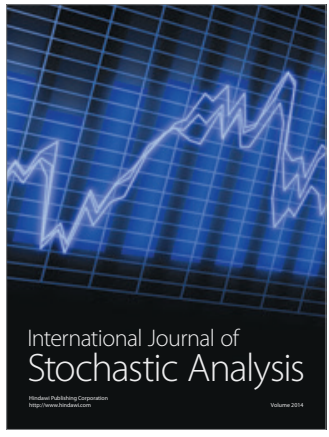

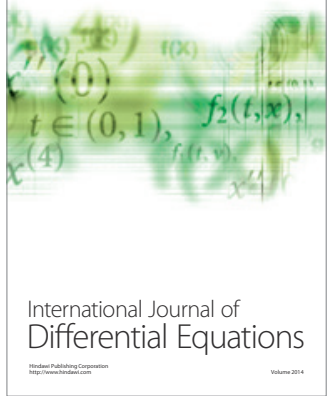
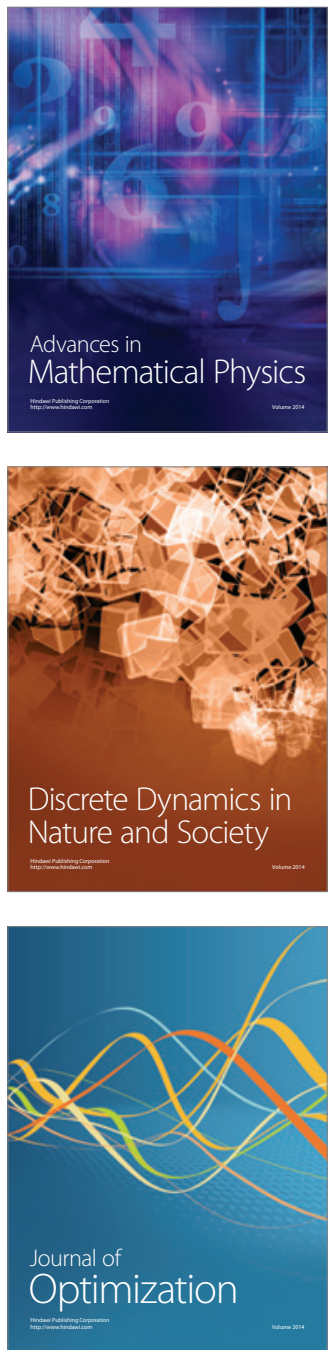